\title{
Hydrogen-bond dynamics for the extended simple point-charge model of water
}

\author{
Francis W. Starr, ${ }^{1, *}$ Johannes K. Nielsen, ${ }^{1,2, \dagger}$ and H. Eugene Stanley ${ }^{1}$ \\ ${ }^{1}$ Center for Polymer Studies, Center for Computational Science, and Department of Physics, Boston University, \\ Boston, Massachusetts 02215 \\ ${ }^{2}$ Department of Mathematics and Physics, University of Roskilde, Postbox 260, DK-4000 Roskilde, Denmark
}

(Received 25 October 1999; revised manuscript received 22 February 2000)

\begin{abstract}
We study hydrogen-bond dynamics in liquid water at low temperatures using molecular dynamics simulations. We analyze the dynamics using energetic and geometric definitions of a hydrogen bond, and employ two analysis methods: (i) a history-dependent correlation function, related to the distribution of bond lifetimes, and (ii) a history-independent correlation function. For method (i) we find an approximately Arrhenius temperature dependence of the bond lifetime, and find that the distribution of bond lifetimes is extremely sensitive to the choice of bond definition. For method (ii) we find-independent of bond definition - that the dynamics are consistent with the predictions of the mode-coupling theory, suggesting that the slow dynamics of hydrogen bonds can be explained in the same framework as standard transport quantities. Our results allow us to clarify the significance of the choice of both bond definition and analysis technique.
\end{abstract}

PACS number(s): 61.43.Fs, 61.20.Ne

\section{INTRODUCTION}

The anomalous properties of water are believed to be connected to the influence of the microscopic behavior of hydrogen bonding on both dynamic and thermodynamic properties of bulk water [1-4]. Both experimental [5-11] and theoretical [12-22] studies have focused on understanding various aspects of the hydrogen bond, including network connectivity and relaxation time, bond lifetime, and other properties. On supercooling, relaxation times typically display a powerlaw behavior with an apparent divergence at a temperature $T_{s} \approx 228 \mathrm{~K}$, that has gained much attention. Molecular dynamics (MD) studies, while limited by the accuracy of the model, are particularly useful for investigating the anomalous behavior in the supercooled regime since nucleation does not occur on the time scale of MD simulations. Furthermore, MD provides immediate access to hydrogen-bond information, which expedites investigation of the bond dynamics. As a result, several studies previously focused on bond dynamics using MD.

Here we investigate the temperature dependence of the hydrogen-bond dynamics using the extended simple point charge (SPC/E) model of water [23]. A brief report of some of our results recently appeared [24], and here we provide a more complete account. We study the bond dynamics using two possible definitions of a hydrogen bond, and consider two analysis methods first proposed by Stillinger [25]: (i) correlations in a time series of bonds which are continuously intact, and thus dependent on the history of bond breaking; and (ii) correlations independent of the history of bondbreaking events.

\footnotetext{
*Present address: Polymers Division and Center for Theoretical and Computational Materials Science, National Institute of Standards and Technology, Gaithersburg, MD 20899.

${ }^{\dagger}$ Present address: Department of Mathematical Modeling, Technical University of Denmark, Building 321, Richard Petersens Square, DK-2800 Lyngby, Denmark.
}

For analysis method (i), we find that the average bond lifetime $\tau_{H B}$, measured by the first breaking time, displays an Arrhenius temperature dependence, as expected from experiments $[5,6]$. For analysis method (ii), we find a nonexponential relaxation of the bond correlations and power-law behavior of correlation time $\tau_{R}$ that can be interpreted using mode-coupling theory (MCT) for the dynamics of supercooled liquids [26].

Previously, the transport properties were shown to be well described by MCT [27,28], suggesting that the bond dynamics may be understood in the same theoretical framework as transport properties. Moreover, the possible relationship between the MCT predictions and the observed power-law behavior of water's dynamics quantities was previously discussed [27-29]. Simulations very close to the MCT transition temperature $T_{c}$, show the expected breakdown of the MCT predictions. Furthermore, the dynamic behavior in this region has also been shown to correlate with the properties of the potential energy surface [30]— which may aid in understanding dynamics below $T_{c}$.

In previous work, authors typically focused on either method (i) [15] or method (ii) $[19,20]$. Differences in the results of these studies may be attributed to the model potential, bonding definition, temperature range, or analysis method considered. Here we clarify the effect of the analysis method and bond definition used. Based on this investigation, we propose that analysis method (ii) - correlations independent of bond breaking-provides more useful results. However, we also recommend that future work should use these techniques only as a supplement to calculations of quantities that are directly observable in experiments, such as the depolarized Raman spectrum.

The paper is organized as follows. In Sec. II, we briefly review the phenomenology of fragile supercooled liquid dynamics that is relevant to the simulations we perform, which are described in Sec. III. In Sec. IV, we present the analysis methods we employ to interpret the simulations, and Sec. V provides a brief summary of the results of previous studies. The main results of this work are described in Sec. VI, which 
is divided into several subsections. Finally, Sec. VII presents a brief discussion and a summary.

\section{DYNAMICS OF SUPERCOOLED LIQUIDS}

In this section, we describe some of the formalisms employed to explain the dynamics of supercooled liquids approaching a glass transition. For a more complete review of glassy behavior, we refer the reader to recent reviews $[3,31-$ 33]. Canonical features of the dynamics of supercooled liquids approaching vitrification include the non-Arrhenius behavior of the relaxation time and the nonexponential decay of correlation functions. A number of theories have been offered to explain these dynamics, such as the entropy-based theory developed by Adam, Gibbs, and Di Marzio [34] and the MCT of Götze and co-workers [26].

\section{A. Entropy-based theory}

We first consider the entropy-based theory, which treats the formation of an ideal glass as a true second-order phase transition [34]. One of the primary results of this theory is the prediction that the relaxation time of the liquid approaching the glass transition obeys the Vogel-Fulcher-Tammann (VFT) form [35]

$$
\tau \sim e^{A /\left(T-T_{0}\right)},
$$

where $T_{0}$ is the temperature of a second-order transition, and is associated with the Kauzmann temperature [3,31], the temperature where the extrapolated entropy of the supercooled liquid is equal to that of the stable crystal. Equation (1) is obtained with the assumption, which we will discuss later for the case of water, that the constant pressure specific heat $C_{P}$ depends inversely on temperature. In addition, Eq. (1) predicts a complete structural arrest of the liquid at $T_{0}$. Experimentally, it is known that the fluid is not completely arrested in the glassy state, and the transition to a glassy state is commonly defined to occur at a temperature $T_{g}$ where the viscosity reaches $10^{12} \mathrm{~Pa}$. Therefore, it is not surprising that fits of, e.g., viscosity [34,36] indicate that $T_{0}<T_{g}$. Close to $T_{g}$, the VFT form sometimes fails, and the relaxation reverts to Arrhenius temperature dependence.

\section{B. Mode-coupling theory}

MCT has been the focus of much recent interest, since MCT may provide a quantitative explanation for observed dynamic anomalies of liquids in a limited temperature regime, particularly the range accessible to MD simulations. MCT was originally developed for atomic liquids, and some extensions were recently made to consider molecular liquids [37]. The idealized form of MCT focuses on the transient trapping of a particle by its near neighbors that occurs occurs as temperature is lowered, commonly known as the cage effect. At low temperatures, movement of a single particle requires collective rearrangement of the neighboring particles, giving rise to greatly increased relaxation times. MCT accounts for this behavior by assuming a nonlinear feedback mechanism controlled by a "memory" function that eventually leads to structural arrest at a temperature $T_{c}$. As a result, the predictions of idealized MCT are expected to be valid

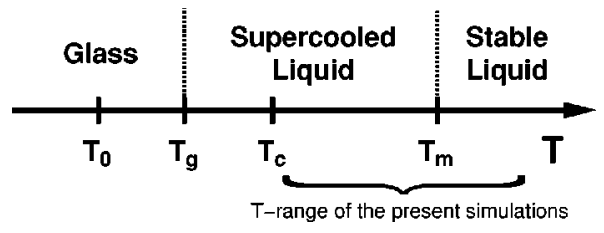

FIG. 1. Relative values for simple liquids of the melting temperature $T_{m}$, and those temperatures often used in discussion of the glass transition: $T_{c}, T_{g}$, and $T_{0} . T_{c}$ is the temperature of structural arrest in MCT, $T_{g}$ is the experimentally defined value of the glass transition, and $T_{0}$ is the temperature that appears in the VFT form of Eq. (1). The predictions of MCT are usually found to be valid in exactly the temperature range that we simulate.

only for $T>T_{c}$ where "activated" processes are not important. These activated processes provide a new mechanism for motion close to $T_{c}$, and thereby a sharp transition is avoided. Fits of data indicate that typically $T_{c} \approx 1.2 T_{g}$ [31]. Figure 1 shows the typical relationship among $T_{m}$ (the melting temperature), $T_{c}$ of MCT, $T_{g}$, and $T_{0}$ of the VFT form.

MCT predicts that any correlation function $c(t)$ related to density fluctuations will decay via a specific two-step process. The first relaxation step is characterized by a rapid decay to a plateau value $c_{p l}$, and near the plateau behaves as a power law:

$$
c(t)-c_{p l} \propto t^{-a} .
$$

The duration of the plateau value is expected to increase as temperature approaches $T_{c}$ from above. The second decay is predicted to behave near the plateau as

$$
c_{p l}-c(t) \propto t^{b}
$$

where $b$, the von Schweidler exponent, typically depends on pressure.

Furthermore, MCT also predicts that the slow relaxation process can be collapsed to a single master curve of the form

$$
\psi(t)=c\left(t / \tau_{R}(T)\right),
$$

where $\tau_{R}(T)$ is the relevant relaxation time. This scaling is frequently referred to as the time-temperature superposition principle (TTSP). MCT predicts that the relaxation times do not follow Arrhenius behavior, but rather conform to a power law

$$
\tau_{R}(T) \sim\left(T-T_{c}\right)^{-\gamma}
$$

where the value of $\gamma$ depends on the thermodynamic path that is followed. The exponents $\gamma, a$, and $b$ are not independent, for only one exponent is needed to predict the values of all exponents. The two equations which determine all the exponents when provided with one value are

$$
\gamma=\frac{1}{2 a}+\frac{1}{2 b}
$$

and

$$
\frac{[\Gamma(1-a)]^{2}}{\Gamma(1-2 a)}=\frac{[\Gamma(1-b)]^{2}}{\Gamma(1+2 b)} \text {. }
$$


The exponents $b$ and $\gamma$ were previously calculated for the SPC/E model [27,28], which we shall compare our results to in Sec. VI.

\section{SIMULATION DETAILS}

We perform MD simulations of 512 water molecules at temperatures $T=350,300,275,250,225,210$, and $200 \mathrm{~K}$, interacting via the SPC/E pair potential [23]. At $T=200 \mathrm{~K}$, we simulate two independent systems, as the long relaxation time at this temperature reduces the quality of time averaging. The SPC/E model treats water as a rigid molecule consisting of three point charges located at the atomic centers of the oxygen and hydrogen, which have an $\mathrm{OH}$ distance of $1.0 \AA$ and a $\mathrm{HOH}$ angle of $109.47^{\circ}$, the tetrahedral angle. Each hydrogen has a charge $q_{H}=0.4238 e$, and the oxygen has a charge $q_{O}=-2 q_{H}$, where $e$ is the fundamental unit of charge. In addition, the oxygen atoms of separate molecules interact via a Lennard-Jones potential with parameters $\sigma$ $=3.166 \AA$ and $\epsilon=0.6502 \mathrm{~kJ} / \mathrm{mol}$. Note that the SPC/E potential displays a density maximum $T_{M D}$ at $T \approx 245 \mathrm{~K}$ at atmospheric pressure [38-40], $32 \mathrm{~K}$ lower than the $T_{M D}$ of $\mathrm{H}_{2} \mathrm{O}$ and $39 \mathrm{~K}$ less than the $T_{M D}$ of $\mathrm{D}_{2} \mathrm{O}$.

We fix the density $\rho=1.0 \mathrm{~g} / \mathrm{cm}^{3}$, and equilibrate all state points to a constant temperature by monitoring the pressure and internal energy. We control the temperature using Berendsen et al.'s method of rescaling the velocities [41], while the reaction field technique with a cutoff of $0.79 \mathrm{~nm}$ [42] accounts for the long-range Coulombic interactions. The equations of motion evolve using the SHAKE algorithm [43] with a time step of $1 \mathrm{fs}$. Equilibration times range from 500 ps at the highest temperature to $15 \mathrm{~ns}$ at the lowest temperature. To analyze the dynamic behavior, it is desirable to make measurements in the isochoric/isoenergetic ensemble (NVE). However, a small energy drift is unavoidable for the long runs presented here, so we again employ the heat bath of Berendsen, using a relaxation time of 200 ps [27]. The large relaxation time prevents an energy drift, but achieves results that are very close to those that would be found were it possible to perform a simulation in the NVE ensemble. For analogous reasons, we choose not to employ constant pressure methods.

\section{ANALYSIS OF SIMULATIONS}

\section{A. The two hydrogen-bond definitions}

We consider two criteria for determining the presence of a hydrogen bond [44]. The definition of Sciortino and Fornili [13], which we will refer to as the energetic bonding criterion, considers two molecules to be bonded if their oxygenoxygen separation is less than $3.5 \AA$ and their interaction energy is less than a threshold energy $E_{H B}$ over a duration exceeding a minimum threshold time. However, we do not include this temporal criterion in our study. We also study a geometric definition $[19,20]$ which uses the same distance criterion but replaces the energetic condition by requiring the $\mathrm{O}-\mathrm{H} \ldots \mathrm{O}$ angle between two molecules to be less than a threshold angle $\theta_{H B}$. We will select the parameters $E_{H B}$ and $\theta_{H B}$ in order to reproduce roughly the experimentally observed temperature dependence of the bond lifetime.

\section{B. The two analysis methods}

We examine the dynamic behavior for each definition using two analysis methods [25]:

(1) Bond autocorrelations for bonds which have remained continuously unbroken (we call these "history-dependent" correlations).

(2) Bond autocorrelations, irrespective of possible bond breaking (we call these "history-independent" correlations).

To formalize the analysis methods, consider a binary operator $h_{i j}(t)$ for each pair of particles $\{i, j\}$, where $h_{i j}(t)$ $=1$ if particle $i$ and $j$ are bonded at time $t$ and $h_{i j}(t)=0$ if $i$ and $j$ are not bonded at time $t[19,20]$. The mean total number of the bonds in the system is given by $\frac{1}{2} N(N-1)\langle h\rangle$, where the brackets denote an average over all possible pairs of molecules and times. The history-independent correlation function $c(t)$ expresses the probability of a randomly chosen pair of molecules being bonded at time $t$, provided that the bond was intact at time $t=0$, independent of possible breaking in the interim time; $c(t)$ is given by

$$
c(t) \equiv \frac{\langle\delta h(0) \delta h(t)\rangle}{\left\langle\delta h^{2}\right\rangle} .
$$

Here $\delta h(t) \equiv h(t)-\langle h\rangle$. In an infinite system, $\langle h\rangle \equiv 0$, since the probability of two arbitrary molecules being bonded is zero. In the finite systems we simulate, the probability of a pair of molecules being bonded is finite, so $\langle h\rangle \neq 0$. We calculate correlations in the fluctuations according to Eq. (7) to eliminate effects of finite $\langle h\rangle$ and compare with expectations of the infinite system.

Luzar and Chandler $[19,20]$ studied the reactive flux, defined by the derivative

$$
k(t) \equiv-\frac{d c(t)}{d t}
$$

which quantifies the probability that an initially present bond breaks at time $t$, independent of possible breaking and reforming events in the interval from 0 to $t$. Thus, the reactive flux measures the effective decay rate of an initial set of hydrogen bonds [45].

For history-dependent correlations, consider Eq. (7), with the restriction that a bond must be continuously intact. As a result, the history-dependent correlation function will decay more rapidly than the history-independent correlation function, as bonds that break and subsequently reform do not contribute to the long-time correlations. For the purposes of our simulations, we will measure distributions of bond lifetimes $P(t)$-in other words, the distribution of first breaking times. Here $P(t)$ measures the probability that an initially bonded pair remains bonded at all times up to $t$ and breaks at time $t$. The function $P(t)$ is obtained from simulations by histogramming the bond lifetimes for each configuration. We note that $P(t)$ is sensitive to the sampling frequency. A choice of a long interval between sampled configurations corresponds to ignoring processes where a bond is broken for a short time and subsequently reforms. As a consequence, to calculate $P(t)$, we sample every $10 \mathrm{fs}$ (shorter than the typical libration time that may destroy a bond). 


\section{PREVIOUS WORK ON BOND DYNAMICS}

There are notable differences between the studies of Sciortino et al. [15] and those of Luzar and Chandler [19,20], which may arise from a variety of reasons related to the difference in bonding definition and different correlation functions considered.

\section{A. Energetic bond definition, history-dependent correlations}

Sciortino et al. calculated $P(t)$ using the energetic bond definition with threshold $E_{H B}=0$ for a system of 216 molecules interacting via the highly structured ST2 potential for water [47]. They considered five temperatures between 350 and $235 \mathrm{~K}$, and found that $P(t)$ does not display an exponential decay, but rather shows a power-law behavior over a range of time, and that this range of behavior extends to longer times at lower temperatures. Furthermore, they found that the average bond lifetime $\tau_{H B}$ shows a power law increase as temperature is lowered, possibly related to the experimental observation that various relaxation times appear to grow with a power-law behavior that diverges at temperature $T_{s} \approx-45^{\circ} \mathrm{C}$.

\section{B. Geometric definition, history-independent correlations}

The study of the reactive flux by Luzar and Chandler $[19,20]$ used the geometric bond definition with a threshold of $\theta_{H B}=30^{\circ}$ for the SPC model of water [46] (which is related to SPC/E) at temperature $T=300 \mathrm{~K}$. Luzar and Chandler observed a nonexponential decay, and further argued that the relaxation of the bond dynamics does not coincide with a power-law decay. They also proposed a model to specifically describe the relaxation of the bond correlations.

\section{RESULTS}

We shall systematically analyze our SPC/E simulation results using both bond definitions and both analysis methods. We first consider the history-dependent correlation function, as the average bond lifetime $\tau_{H B}$ provides and important check of the bond definitions. We then focus on a historyindependent correlation function, and interpret our results using MCT.

\section{A. History-dependent bond correlations}

\section{Average bond lifetime $\tau_{H B}$}

We first calculate $\tau_{H B}$ using threshold values $E_{H B}=$ $-10 \mathrm{~kJ} / \mathrm{mol}$ and $\theta_{H B}=30^{\circ}$ (Fig. 2). Our results are summarized in Table I. We find an Arrhenius temperature dependence with activation energies

$E_{A}= \begin{cases}8.8 \pm 1.0 \mathrm{~kJ} / \mathrm{mol} & (\text { energetic bond definition) } \\ 9.3 \pm 1.0 \mathrm{~kJ} / \mathrm{mol} & \text { (geometric bond definition), }\end{cases}$

which compare well with the activation energy $E_{A}=10.8$ $\pm 1.0 \mathrm{~kJ} / \mathrm{mol}$ obtained from depolarized light scattering experiments $[5,6]$. Indeed, we chose the thresholds of the bonding criteria so that we roughly reproduce the activation energy obtained from experimental measurements. We also observe an Arrhenius behavior for threshold values $E_{H B}$

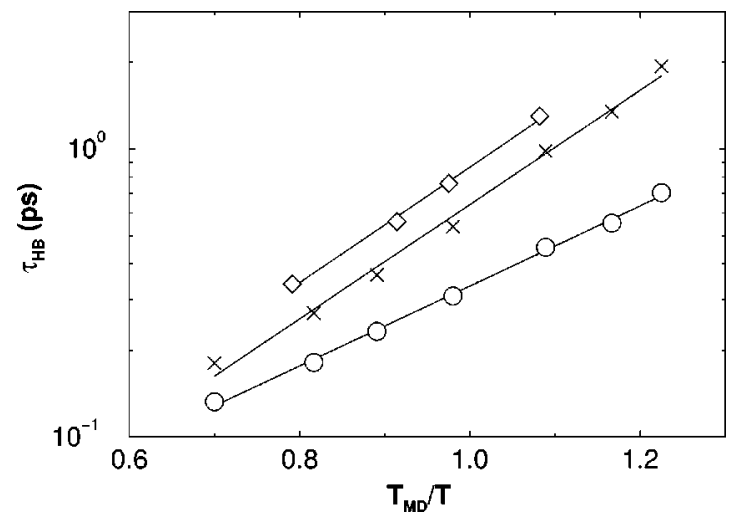

FIG. 2. Average bond lifetime $\tau_{H B}$ for the energetic $(\bigcirc)$ and geometric $(X)$ bond definitions, shown for comparison are experimental data $(\diamond)$ for depolarized light scattering [5]. Although the experimental data and the results for the geometric definition both display a weak upward curvature, all three curves can be fit by Arrhenius behavior $\tau_{H B}=\tau_{0} \exp \left(E_{A} / k T\right)$. We scale the temperature of the simulation results by $T_{M D}^{S P C / E} \approx 245 \mathrm{~K}$ [39], and temperature of the experimental data by $T_{M D}^{\text {expt }}=277 \mathrm{~K}$ to facilitate comparison [38-40].

$=0 \mathrm{~kJ} / \mathrm{mol}[13,15]$ and $\theta_{H B}=35^{\circ}$. For these parameters, we find the $E_{A}$ is roughly $30 \%$ smaller using the energetic definition, and is roughly $10 \%$ smaller using the geometric definition. The activation energy $E_{A}$ associated with $\tau_{H B}$ was interpreted as the energy required to break a bond via librational motion, a "fast" motion [5,6]. We find better quantitative agreement with experiments for $\tau_{H B}$ values obtained from the geometric definition than for $\tau_{H B}$ values obtained from energetic definition-possibly because the geometric bond definition, like the results of the depolarized light scattering experiments $[5,6]$, is highly sensitive to the linearity of the bond. We will use the thresholds $E_{H B}=-10 \mathrm{~kJ} / \mathrm{mol}$ and $\theta_{H B}=30^{\circ}$ for the remaining calculations.

\section{Distribution $P(t)$ of bond lifetimes}

We now turn our attention to the distribution $P(t)$ of bond lifetimes. We observe neither power-law nor exponential behavior of $P(t)$ for small times (Fig. 3). However the tail of the distribution is well approximated by an exponential. A power-law behavior of $P(t)$ was found in Ref. [15] by studying the ST2 model, using an energetic bond definition with a threshold parameter $E_{H B}=0 \mathrm{~kJ} / \mathrm{mol}$, and the previously mentioned temporal requirement. The difference between the

TABLE I. Average hydrogen-bond lifetime $\tau_{H B}$ using each bond definition.

\begin{tabular}{ccc}
\hline \hline \multicolumn{3}{c}{$\tau_{H B}(\mathrm{ps})$} \\
$T(\mathrm{~K})$ & Energetic bond definition & Geometric bond definition \\
\hline 350 & 0.13 & 0.18 \\
300 & 0.18 & 0.27 \\
275 & 0.23 & 0.37 \\
250 & 0.31 & 0.54 \\
225 & 0.46 & 0.99 \\
210 & 0.55 & 1.35 \\
200 & 0.71 & 1.94 \\
\hline \hline
\end{tabular}




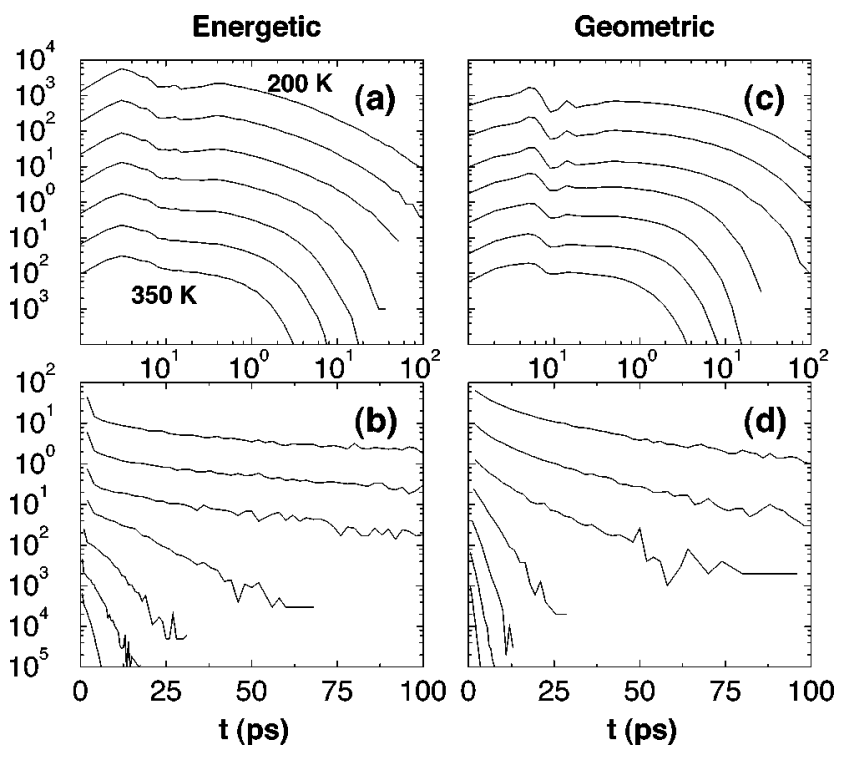

FIG. 3. The history-dependent bond lifetime distribution $P(t)$ for the energetic bond definition plotted on (a) a log-log scale and (b) a log-linear scale, revealing an exponential tail. Also shown is $P(t)$ for the geometric bond definition on (c) a log-log scale and (d) a log-linear scale, also consistent with an exponential tail. Reading from top to bottom, the curves may be identified as $T=200,210$, $225,250,275,300$, and $350 \mathrm{~K}$. Each curve is offset by one decade for clarity.

present findings for $P(t)$ and those of Ref. [15] is likely attributable a combination of two factors: (i) including a temporal criterion more closely links the dynamics to diffusive motion, which is typically described by a power law; and (ii) that Ref. [15] followed a path very near to the liquid-liquid critical point that appears in the ST2 potential [48]. If a liquid-liquid transition occurs in SPC/E, it has been estimated to terminate at a critical point located at roughly 160 $\mathrm{K}$ and $200 \mathrm{MPa}$ [39]. The present simulations should not be significantly affected by this critical point, since the temperature and pressure we simulate are far from it. Simulations at lower temperature, which are not feasible with current computational resources, may help to resolve this question.

The small difference between the results from the two bond definitions can be explained by the difference in sensitivity to librational motion. Specifically, the sensitivity of the geometric definition to librations causes many bonds to break on a short time scale. Since $P(t)$ depends on the unbroken presence of the bond, short time fluctuations result in quantitative and qualitative differences in the functional form of $P(t)$.

\section{B. History-independent bond correlations}

We next consider the history-independent bond correlation function $c(t)$. We calculate $c(t)$ by averaging over all pairs in the system and many possible time origins. Our results for all temperatures and both bond definitions are shown in Figs. 4(a) and 4(b). For $t \leqq 0.1 \mathrm{ps,} c(t)$ decays rapidly due to librational motion. For $t \geq 0.1 \mathrm{ps}, c(t)$ remains relatively constant, especially at low $T$. Following the "plateau" is a second decay region where the correlations relax to zero. This two-step relaxation is typical of super-
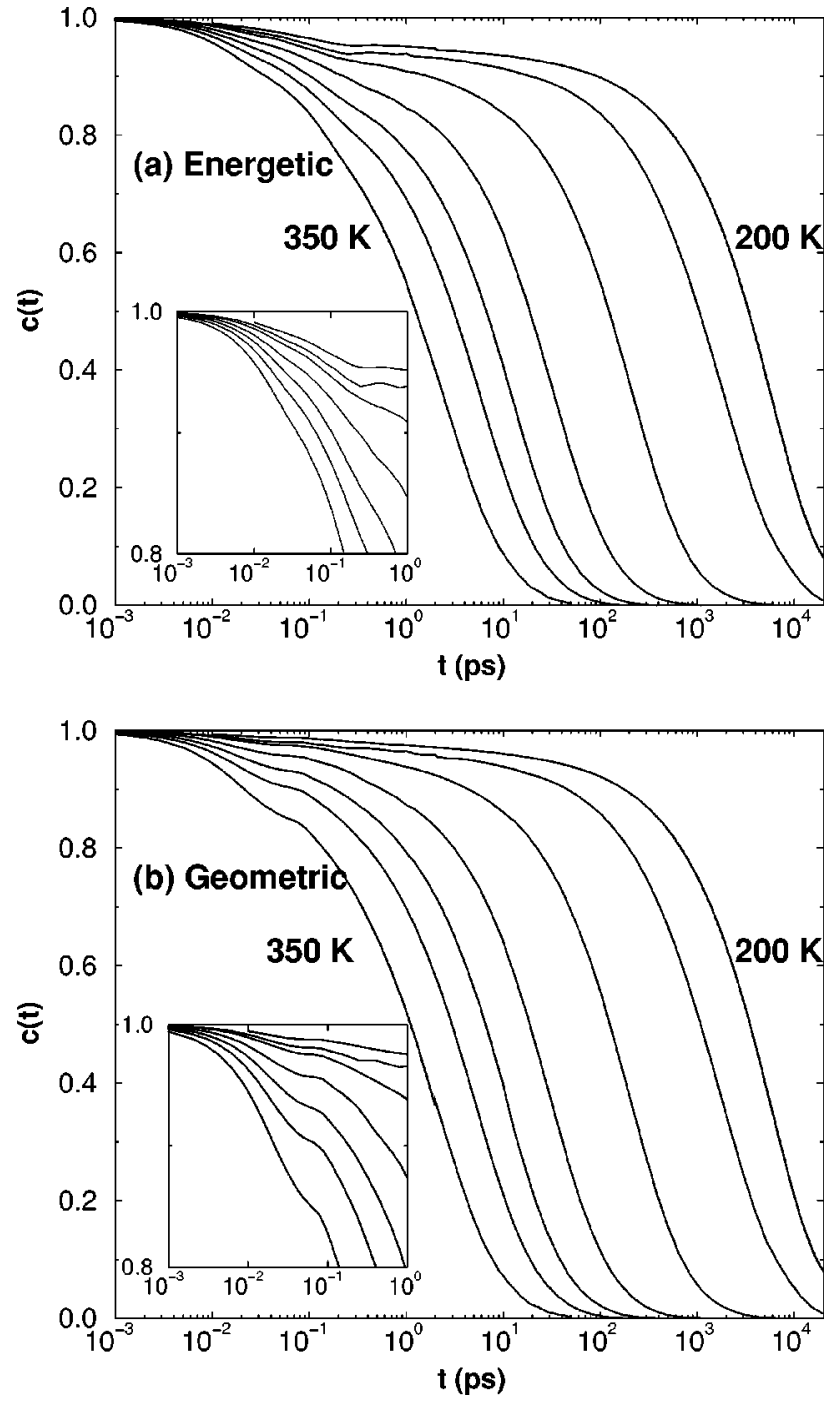

FIG. 4. For the history-independent method, we show the decay of the hydrogen-bond correlation function $c(t)$ for both bond definitions; (a) the energetic bond definition and (b) the geometric bond definition. The simulations are for temperatures $T=350,300,275$, $250,225,210$, and $200 \mathrm{~K}$. Since the two definitions are nearly identical for $t>1 \mathrm{ps}$, we show an enlargement of the data in the region $t \leqslant 1 \mathrm{ps}$ in the inset.

cooled liquids [3,31-33]. In the second decay region, the dominant process for bond breaking is diffusion. Since both bond definitions employ the same distance criterion, it is not surprising that the decay of $c(t)$ at large times is nearly identical for each of the two bond definitions. The decay of $c(t)$ is very similar to that observed for the intermediate scattering function [27,28]. Indeed, both these functions show a rapid decrease at small times due to vibrations and librations of molecules, followed by a slow decay that can be described by a stretched exponential. The intermediate scattering function is a measure of the correlation of the molecular coordinates, while $c(t)$-at least in the long time limit-is a measure of the correlation of the molecular coordinates between a pair which is initially bonded. Hence it is not surprising that the functions are qualitatively similar. Indeed, both functions are controlled by the diffusivity of the system for long time scales. 


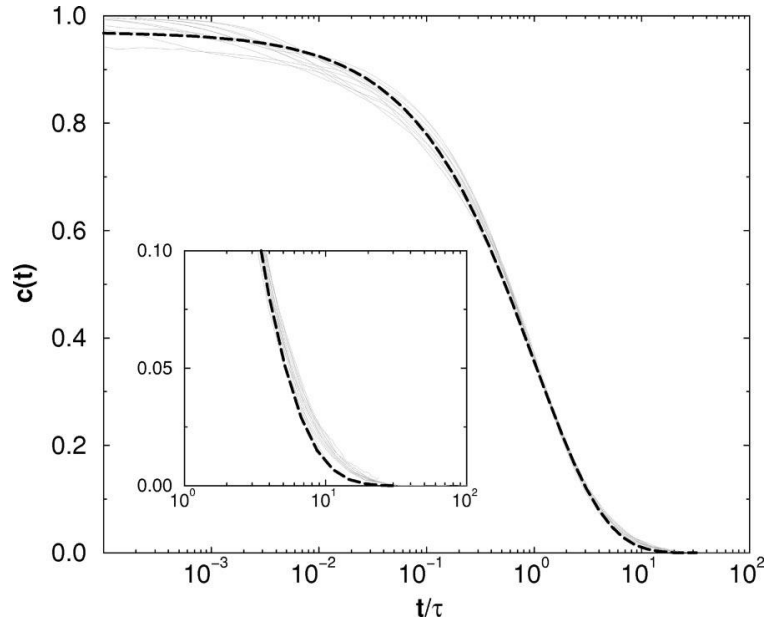

FIG. 5. Data collapse of $c(t)$ using the scaling form predicted by MCT. The time has been rescaled by $\tau_{R}$ as defined in Eq. (11). Also shown is the fit using Eq. (10) (dashed line). Note the small deviation from the fit for $t / \tau \gtrsim 5$ (see the inset).

\section{Scaling of the correlation function}

We may interpret the relaxation shown in Fig. 4 in terms of the predictions of MCT. We first consider the TTSP that focuses on the scaling of the second relaxation regime. Previous workers tested the scaling form of Eq. (4), and found reasonable collapse to a single master curve at long times for sufficiently low temperatures $[27,49]$. We obtain a reasonable collapse of the all $c(t)$ curves (for both definitions) using Eq. (4) (Fig. 5), and fit with the Kohlrausch-WilliamWatts stretched-exponential

$$
c\left(t / \tau_{R}(T)\right)=A_{0} \exp \left[-\left(t / \tau_{R}\right)^{\beta}\right],
$$

where $\beta=0.66 \pm 0.06$ and $A_{0}=0.97 \pm 0.05$. The fit by Eq. (10) deviates slightly from the simulation results for $t / \tau_{R}$ $\geq 5[50]$. We note that each curve may also be individually fit by a stretched exponential prior to scaling, from which we obtain $\beta$ values in the range $0.60-0.73$.

\section{Temperature dependence of relaxation time}

We define the relaxation time $\tau_{R}$ by

$$
c\left(t=\tau_{R}\right)=e^{-1} .
$$

Our results for $\tau_{R}$ are given in Table II. In accord with Eq. (5), we plot $\tau_{R}$ as a function of $\left(T-T_{c}\right)$. We find, indepen-

TABLE II. Relaxation time $\tau_{R}$ of the correlation function $c(t)$ defined by Eq. (11).

\begin{tabular}{ccc}
\hline \hline & \multicolumn{2}{c}{$\tau_{R}(\mathrm{ps})$} \\
$T(\mathrm{~K})$ & Energetic bond definition & Geometric bond definition \\
\hline 350 & 2.41 & 2.06 \\
300 & 5.62 & 5.18 \\
275 & 11.5 & 10.9 \\
250 & 33.0 & 32.1 \\
225 & 214 & 213 \\
210 & 1839 & 1857 \\
200 & 6174 & 6239 \\
\hline \hline
\end{tabular}

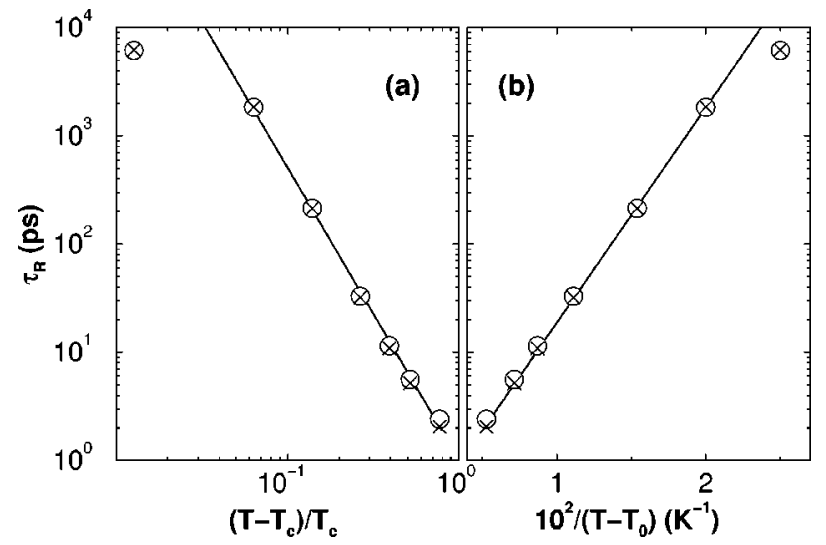

FIG. 6. Relaxation time $\tau_{R}$ of the hydrogen-bond correlation function $c(t)$ (Table II). (a) Fit to the scaling form predicted by mode-coupling theory with $T_{c}=197.5 \mathrm{~K}$. (b) Fit to the empirical VFT form with $T_{0}=160 \mathrm{~K}$. The symbols represent the relaxation time from the geometric $(X)$ and energetic $(O)$ bond definitions. The deviation from both fitting forms may indicate a smooth transition of relaxations time in supercooled water with that of glassy water.

dent of bond definition, a power-law behavior with $T_{c}$ $=197.5 \pm 2 \mathrm{~K}$ and $\gamma=2.7 \pm 0.1$ for $T \geqslant 210 \mathrm{~K}$ [Fig. 6(a)]. These values were independently measured using the diffusion constant in Ref. [28] for SPC/E, and were found to be $\gamma=2.8$ and $T_{c}=194 \mathrm{~K}$. Our results for $\tau_{R}$ may also be relatively well fit by these parameters. The minor difference between the values of $T_{c}$ may arise from the decoupling of relaxation times and diffusion constant that is known to occur at low $T[28,51]$. At $T=200 \mathrm{~K}, \tau_{R}$ is smaller than would be estimated by Eq. (5), most likely because MCT does not account for activated processes which aid diffusion and reduce relaxation times at low temperatures. Typically, these activated processes become important near $T_{c}$, as we find. Calculations of the diffusion constant and the relaxation time of the intermediate scattering function also show deviations from a power-law at $T=200 \mathrm{~K}$. Thus the discussion of $\tau_{R}$ appears to apply equally well to these other quantities.

Our results for $\tau_{R}$ can also be fit by the VFT form of Eq. (1) where $T_{0}=160 \mathrm{~K}$ [Fig. 6(b)]. As discussed in Sec. II, the VFT form is derived assuming that the constant pressure specific heat $C_{P}$ depends inversely on the absolute temperature. For water, $C_{P}$ is known to be well approximated by a power law with a nonzero singular temperature [2]. Thus it is somewhat surprising that our results may be fit well by the VFT.

\section{Reactive flux}

The behavior of the reactive flux $k(t)$ is shown in Figs. 7 (a) and 7(b) for both bond definitions. We observe a powerlaw region starting at $t \approx 0.3 \mathrm{ps}$ for both bond definitions, with an exponent $-0.5 \pm 0.1$ for temperatures down to $T$ $=250 \mathrm{~K}$. In addition, the duration of the power-law region increases from about 1.5 decades at $300 \mathrm{~K}$ to about two decades at $250 \mathrm{~K}$. The value of this exponent can also be interpreted in terms of MCT predictions. In the region of the power law of Fig. 7, Eq. (3) is valid. Since the plateau region 


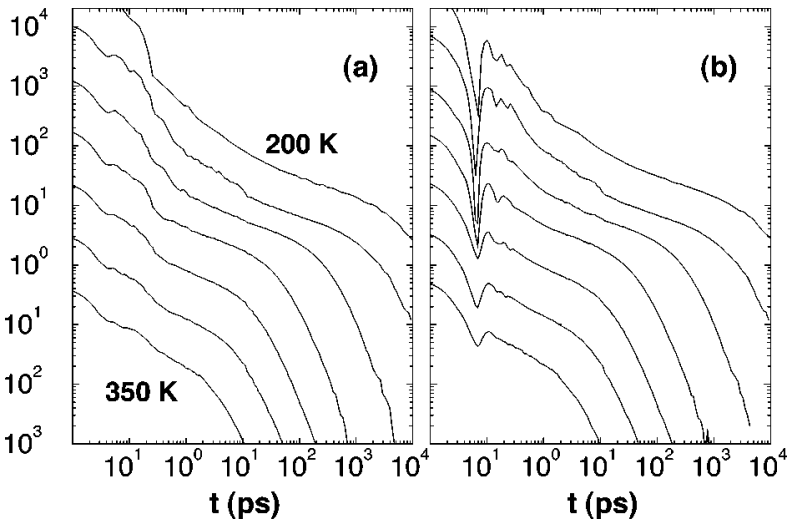

FIG. 7. The reactive flux $k(t)$ for (a) the energetic bond definition and (b) the geometric bond definition. These curves show little resemblance to $P(t)$ plotted in Fig. 3, as discussed in the text. Each curve is offset by one decade for clarity. The temperatures shown are the same as in Fig. 2. Note that the results obtained for the geometric definition at $T=300 \mathrm{~K}$ are consistent with the calculations of Luzar and Chandler for the SPC potential (see the inset of Fig. 1 of Refs. [19] and [20]).

of $c(t)$ is difficult to identify unambiguously, studying the derivative expedites identification of $b$, as Eqs. (3) and (8) require

$$
k(t) \propto t^{b-1}
$$

Thus $b=0.5 \pm 0.1$. For $T<250 \mathrm{~K}$, our results may be fit by Eq. (3) if higher-order terms are included. To properly measure $b$, a detailed analysis of transport properties is needed, such as that performed in Refs. [27,28]. However, our purpose is not to confirm the MCT predictions, but rather to determine if the bond dynamics can be interpreted within the MCT framework. Our results support this possibility, since $\gamma=2.7$ and $b=0.5$ are consistent with the MCT predictions of Eqs. (6a) and (6b).

We note that the reactive flux for the geometric bond definition [Fig. 7(a)] shows a dip at $t \approx 0.06$ ps not present in the reactive flux obtained from the energetic bond definition [Fig. 7(b)]. The difference can be reconciled by considering the sensitivity of each definition to librational motion. The angular restriction of the geometric bond definition makes it very sensitive to librational motion, which has a characteristic time below $0.1 \mathrm{ps}$, so the dip stems from pairs initially bonded which are broken (by libration) but often reform a bond. In contrast, the energetic definition is far less sensitive to bond bending $[13,15]$, so we observe a relatively flat behavior at early times. At long times, $k(t)$ is independent of bond definition, as expected since both definitions use the same distance criterion.

\section{DISCUSSION}

We presented results for the hydrogen-bond dynamics using two analysis techniques for the SPC/E potential, which allows us to effectively address the differences obtained by each method. In particular, we contrast the functions $P(t)$ and $k(t) ; P(t)$ measures the probability of an initially present bond to first break at time $t$, while $k(t)$ measure the decay rate of a bond, allowing reforming events. The signifi-

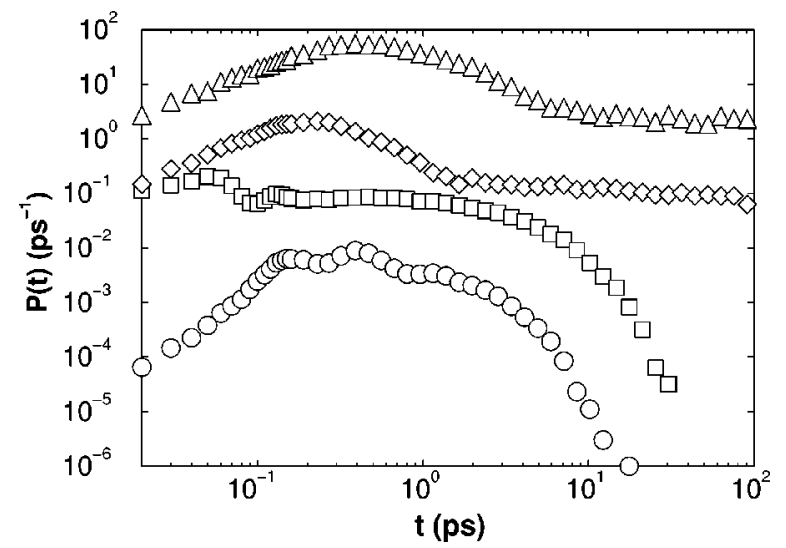

FIG. 8. The bond lifetime distribution for the geometric definition with various bond angles at $T=250 \mathrm{~K}$. The angles considered are $15^{\circ}(\bigcirc), 30^{\circ}(\square)$ (the case of Fig. 2$), 45^{\circ}(\diamond)$, and $60^{\circ}(\triangle)$. Each curve is offset by 1.5 decades for clarity.

cant difference in the behavior of $P(t)$ (Fig. 3) relative to $k(t)$ (Fig. 7) demonstrates that exponential behavior in the history-dependent $P(t)$ does not imply a specific behavior in history-independent $c(t)$ and $k(t)$, contradicting the previous claim that $c(t)$ and $k(t)$ decay exponentially if and only if the lifetime distribution is also exponential [19]. This is best illustrated by the fact that for the $t \gtrsim 5 \mathrm{ps}, P(t)$ is indeed well approximated by an exponential [Figs. 3(b) and $3(\mathrm{~d})$ ].

The differences in the qualitative behavior of $P(t)$ for the two bond definitions (Fig. 3) suggest that $P(t)$ may not be the optimal function for understanding the bond dynamics. To emphasize the difficulties of this approach, we plot $P(t)$ for several possible bond-angle criteria for the geometric bond definition at $T=250 \mathrm{~K}$ (Fig. 8). All curves are significantly different from one another, further illustrating that the behavior of $P(t)$ is strongly dependent on the choice of bonding criteria. In contrast, the results of the historyindependent $c(t)$ and $k(t)$ appear to be largely independent of the bond definition at long times.

The long time behavior of the history-independent correlations is not strongly affected by the choice of bonding definition, provided a reasonable distance criterion is chosen. The short time behavior is sensitive to the librational motion of the molecules, and as a result shows a strong dependence on bond definition. The scaling of the correlation function indicates that the relaxation obeys a single master relaxation that is temperature independent over the range of temperature studied.

The exponent values $\gamma=2.7$ and $b=0.5$ obtained from the history-independent analysis are close to the values $\gamma$ $=2.80$ and $b=0.51$ in the MCT analysis along the same thermodynamic path [28]. Hence our results are consistent with the slowing of the dynamics as described by MCT, with a "dynamical critical temperature", $T_{c}$ about $50 \mathrm{~K}$ below $T_{M D}$ for the path studied. Since the dynamics of typical transport properties have already been shown to be consistent with MCT [27,28], it appears that the same explanation may be used for the bond dynamics studied here as used for the typical transport quantities. In water, this may not be the case, since our bond definitions do not consider the quantum effects that arise from the sharing of a proton. Furthermore, as mentioned in Sec. I, our results may not be directly tested 
experimentally; therefore, we suggest the further simulation work should consider comparisons with experimentally measured quantities, such as the Raman spectrum, so that the significance of these somewhat arbitrary definitions can be better understood.

Attempts to explain the experimentally measured thermodynamic and dynamic anomalies have led to much scrutiny of the phase diagram in the supercooled regime. Previous work emphasized that the power-law growth of relaxation time, and the fact that location of the mode coupling $T_{c}$ coincides with $T_{s}$ is sufficient to account for the observed dynamic anomalies. This explanation is consistent with the hypothesis that the liquid and glass are continuous, without the intervention of a low temperature spinodal [52]. The deviation of characteristic times at the lowest temperature we study provides further support for continuity of the liquid and glassy states of water. However, we emphasize that the MCT explanation does not account for the unusual behavior of thermodynamic properties. Further investigations may help to elucidate the connection, if any, with a possible additional critical point in the supercooled region of the phase diagram $[4,48,53]$, or possibly the "singularity-free" scenario [54].

The correlation function $c(t)$ yields information about the relation between molecule pair separation and orientation, as these two properties are used to define the hydrogen bond. Similar information can be obtained by considering angular and diffusion correlations; indeed, the dipole autocorrelation function and the intermediate scattering function for SPC/E at roughly the same state points reveal similar information [27]. To obtain bond information that is not as tightly coupled to the classical dynamics of the molecules, consideration of flexibility, polarizability, and the quantum mechanical aspects of the water molecule is needed. This is an area of interesting future investigation, as the results would provide the validity of classical results.
The Arrhenius behavior of the average bond lifetime and the power-law behavior of the relaxation time are not unique to water. Thermally activated bond lifetimes coupled with slow network restructuring have been found in a hard-sphere model with a restricted coordination number [55]. This suggests that the dynamics observed in here may also apply to other networked fluids, such as $\mathrm{SiO}_{2}$.

A more complete picture of the dynamics in liquid water may be provided by investigating history-independent correlations for the ST2 model of water, which is more structured and also more mobile than SPC/E water, and would thus provide a contrast to the results presented here. In ST2, the presence of activated processes for $T \$ 270 \mathrm{~K}$ yield Arrhenius behavior of the diffusion constant, with an activation energy of $115 \mathrm{~kJ} / \mathrm{mol}$ [56]. These differences further illustrate the sensitivity of the dynamics on the model potential, most of which provide a good account of structural properties. Furthermore, simulations of the dynamic properties of the TIP4P [57] potential have been considered only in a limited range, and further studies would be useful. Previous work suggests that the behavior of liquid water is frequently bounded by the behavior seen in the ST2, TIP4P, and SPC/E models of water [39]. Studies of the spatial correlations of the dynamically active regions [58] are also underway for these potentials. Combining simulation results for many potentials could provide more insight into the hydrogen bonds dynamics in supercooled liquid water.

\section{ACKNOWLEDGMENTS}

We wish to thank C.A. Angell, S.V. Buldyrev, D. Chandler, S. Havlin, A. Luzar, S. Sastry, F. Sciortino, A. Skibinsky, and R.J. Speedy for helpful discussions. All simulations were performed using the Boston University 192-processor SGI/Cray Origin 2000 supercomputer. The Center for Polymer Studies is supported by the NSF.
[1] D. Eisenberg and W. Kauzmann, The Structure and Properties of Water (Oxford University Press, New York, 1969).

[2] C. A. Angell, in Water: A Comprehensive Treatise, edited by F. Franks (Plenum, New York, 1981); Annu. Rev. Phys. Chem. 34, 593 (1983).

[3] P. G. Debenedetti, Metastable Liquids (Princeton University Press, Princeton, 1996).

[4] O. Mishima and H. E. Stanley, Nature (London) 396, 329 (1998).

[5] S.-H. Chen and J. Teixeira, Adv. Chem. Phys. 64, 1 (1985).

[6] C. J. Montrose, J. A. Bucaro, J. Marshall-Coakley, and T. A. Litovitz, J. Chem. Phys. 60, 5025 (1974); W. Danninger and G. Zundel, ibid. 74, 2769 (1981); O. Conde and J. Teixeira, J. Phys. (France) 44, 525 (1983); Mol. Phys. 53, 951 (1984); Y. Suzuki, A. Fujiwara, and Y. Tominaga (unpublished).

[7] D. Bertolini, M. Cassettari, and G. Salvetti, J. Chem. Phys. 76, 3285 (1982).

[8] S.-H. Chen, J. Teixeira, and R. Nicklow, Phys. Rev. A 26, 3477 (1982).

[9] C. A. Angell and V. Rodgers, J. Chem. Phys. 80, 6245 (1984).

[10] J. D. Cruzan, L. B. Braly, K. Liu, M. G. Brown, J. G. Loeser, and R. J. Saykally, Science 271, 59 (1996).

[11] S. Woutersen, U. Emmerichs, and H. Bakker, Science 278, 658 (1997).

[12] A. Geiger, P. Mausbach, J. Schnitker, R. L. Blumberg, and H. E. Stanley, J. Phys. (Paris), Colloq. 45, C7-13 (1984).

[13] F. Sciortino and S. L. Fornili, J. Chem. Phys. 90, 2786 (1989).

[14] D. Bertolini et al., J. Chem. Phys. 91, 1179 (1989); 91, 1191 (1989).

[15] F. Sciortino, P. H. Poole, H. E. Stanley, and S. Havlin, Phys. Rev. Lett. 64, 1686 (1990).

[16] A. Luzar and D. Chandler, J. Chem. Phys. 98, 8160 (1993).

[17] P. H. Poole, F. Sciortino, T. Grande, H. E. Stanley, and C. A. Angell, Phys. Rev. Lett. 73, 1632 (1994).

[18] R. Lamanna, G. Floridi, and S. Cannistraro, Phys. Rev. E 52, 4529 (1995).

[19] A. Luzar and D. Chandler, Nature (London) 379, 55 (1996).

[20] A. Luzar and D. Chandler, Phys. Rev. Lett. 76, 928 (1996).

[21] M. Matsumoto and I. Ohmine, J. Chem. Phys. 104, 2705 (1996).

[22] E. Shiratani and M. Sasai, J. Chem. Phys. 104, 7671 (1996)

[23] H. J. C. Berendsen, J. R. Grigera, and T. P. Stroatsma, J. Phys. Chem. 91, 6269 (1987). 
[24] F. W. Starr, J. K. Nielsen, and H. E. Stanley, Phys. Rev. Lett. 82, 2294 (1999).

[25] F. H. Stillinger, Adv. Chem. Phys. 31, 1 (1975).

[26] W. Götze and L. Sjögren, Rep. Prog. Phys. 55, 241 (1992); W. Götze, in Liquids, Freezing and the Glass Transition, edited by J. P. Hansen, D. Levesque, and J. Zinn-Justin (North-Holland, Amsterdam, 1991).

[27] P. Gallo, F. Sciortino, P. Tartaglia, and S.-H. Chen, Phys. Rev. Lett. 76, 2730 (1996); F. Sciortino, P. Gallo, P. Tartaglia, and S.-H. Chen, Phys. Rev. E 54, 6331 (1996); S.-H. Chen, P. Gallo, F. Sciortino, and P. Tartaglia, ibid. 56, 4231 (1997); F. Sciortino, L. Fabbian, S.-H. Chen, and P. Tartaglia, ibid., 56, 5397 (1997).

[28] F. W. Starr, S. Harrington, F. Sciortino, and H. E. Stanley, Phys. Rev. Lett. 82, 3629 (1999); F. W. Starr, F. Sciortino, and H. E. Stanley, Phys. Rev. E 60, 6757 (1999).

[29] F. X. Prielmeier, E. W. Lang, R. J. Speedy, and H.-D. Lüdemann, Phys. Rev. Lett. 59, 1128 (1987).

[30] E. La Nave, A. Scala, F. W. Starr, F. Sciortino, and H. E. Stanley, Phys. Rev. Lett. 84, 4605 (2000); A. Scala, F. W. Starr, E. La Nave, F. Sciortino, and H. E. Stanley, Nature (London) (to be published).

[31] C. A. Angell, Science 267, 1924 (1995).

[32] F. H. Stillinger, Science 267, 1935 (1995).

[33] W. Kob, in Supercooled Liquids, edited by J. T. Fourkas, D. Kivelson, U. Mohanty, and K. A. Nelson (American Chemical Society, Washington, DC, 1997), pp. 28-44, and references contained therein.

[34] G. Adam and J. H. Gibbs, J. Chem. Phys. 43, 139 (1965); J. H. Gibbs and E. A. Di Marzio, ibid. 28, 373 (1958).

[35] H. Vogel, Z. Phys. 22, 645 (1921); G. S. Fulcher, J. Am. Ceram. Soc. 8, 339 (1925); G. Tammann and W. Z. Hesse, Anorg. Allgem. Chem. 156, 245 (1926); G. W. Scherer, J. Am. Ceram. Soc. 75, 1060 (1992).

[36] C. A. Angell and K. J. Rao, J. Chem. Phys. 57, 470 (1972); C. A. Angell and J. C. Tucker, J. Phys. Chem. 78, 278 (1974); C. A. Angell, D. R. MacFarlane, and M. Oguni, in Dynamical Aspects of Structural Change in Liquids and Glasses, edited by C. A. Angell and M. Goldstein (Ann. N.Y. Academy of Science, New York, 1984), p. 241.

[37] R. Schilling and T. Scheidsteger, Phys. Rev. E 56, 2932 (1997); T. Franosch, M. Fuchs, W. Götze, M. R. Mayr, and A. P. Singh, ibid. 56, 5659 (1997); L. Fabbian, F. Sciortino, F. Thiery, and P. Tartaglia, ibid. 57, 1485 (1998); L. Fabbian, A. Latz, R. Schilling, F. Sciortino, P. Tartaglia, and C. Theis, ibid. 60, 5768 (1999).

[38] L. Baez and P. Clancy, J. Chem. Phys. 101, 8937 (1994).

[39] S. Harrington, P. H. Poole, F. Sciortino, and H. E. Stanley, J. Chem. Phys. 107, 7443 (1997).

[40] K. Bagchi, S. Balasubramanian, and M. L. Klein, J. Chem. Phys. 107, 8651 (1997).

[41] H. J. C. Berendsen, J. P. M. Postma, W. F. van Gunsteren, A. DiNola, and J. R. Haak, J. Chem. Phys. 81, 3684 (1984).
[42] O. Steinhauser, Mol. Phys. 45, 335 (1982).

[43] J.-P. Ryckaert, G. Ciccotti, and H. J. C. Berendsen, J. Comput. Phys. 23, 327 (1977).

[44] To properly simulate hydrogen-bond dynamics in liquid water, it is necessary to include the quantum effects which lead to the sharing of a proton between two oxygens. However, numerous MD studies have shown that simple two-body classical potentials give the correct qualitative static and dynamic features of water. Including additional effects would change the quantitative results presented, and might alter the conclusions drawn from these results.

[45] D. Chandler, Introduction to Modern Statistical Mechanics (Oxford University Press, Oxford, 1987).

[46] H. J. C. Berendsen, J. P. M. Postma, W. F. van Gusteren, and J. Hermans, in Intermolecular Forces, edited by B. Pullman (Reidel, Dordrecht, 1981), p. 331.

[47] F. H. Stillinger and A. Rahman, J. Chem. Phys. 60, 1545 (1974).

[48] P. H. Poole, F. Sciortino, U. Essmann, and H. E. Stanley, Nature (London) 360, 324 (1992); Phys. Rev. E 48, 3799 (1993); F. Sciortino, P. H. Poole, U. Essmann, and H. E. Stanley, ibid. 55, 727 (1997); S. Harrington, R. Zhang, P. H. Poole, F. Sciortino, and H. E. Stanley, Phys. Rev. Lett. 78, 2409 (1997).

[49] W. Kob and H. C. Andersen, Phys. Rev. Lett. 73, 1376 (1994); Phys. Rev. E 51, 4626 (1995); 52, 4134 (1995).

[50] The deviation of the tail from the stretched exponential has a variety of possible explanations. It is possible that the decay of $c(t)$ does not coincide with a stretched exponential. Alternatively, the deviation may arise from insufficient statistics or a finite size effect, possibilities currently being studied.

[51] S. C. Glotzer, V. N. Novikov, and T. B. Schrøder, J. Chem. Phys. 112, 509 (2000).

[52] R. J. Speedy and C. A. Angell, J. Chem. Phys. 65, 851 (1976); R. J. Speedy, ibid. 86, 982 (1982).

[53] O. Mishima and H. E. Stanley, Nature (London) 392, 192 (1998); H. Tanaka, Nature (London) 380, 328 (1996); J. Chem. Phys. 105, 5099 (1996); Y. Katayama, et al., Nature (London) 403, 170 (2000); O. Mishima, Phys. Rev. Lett. (to be published).

[54] H. E. Stanley and J. Teixeira, J. Chem. Phys. 73, 3404 (1980); S. Sastry, P. G. Debenedetti, F. Sciortino, and H. E. Stanley, Phys. Rev. E 53, 6144 (1996).

[55] R. J. Speedy, Mol. Phys. 89, 1121 (1996); R. J. Speedy and P. G. Debenedetti, ibid. 83, 591 (1995).

[56] D. Paschek and A. Geiger, J. Phys. Chem. B 103, 4139 (1999).

[57] W. L. Jorgensen, J. Chandrasekhar, J. Madura, R. W. Impey, and M. L. Klein, J. Chem. Phys. 79, 926 (1983).

[58] W. Kob, C. Donati, S. J. Plimpton, and S. C. Glotzer, Phys. Rev. Lett. 79, 2827 (1997); C. Donati, J. F. Douglas, W. Kob, S. J. Plimpton, P. H. Poole, and S. C. Glotzer, ibid. 80, 2338 (1998). 